\title{
Tratamiento de adenocarcinoma prostático localizado en paciente con trasplante renal mediante Ultrasonido de Alta Intensidad
}

\author{
E. Lledó García, J. Jara Rascón, J.Mํㅡㄹ Díez Cordero, D. Subirá Ríos, A. Alvarado*, \\ F. Anaya Fernández de Lomana **, C. Hernández Fernández \\ Servicios de Urología, *Anatomía Patológica ${ }^{* *}$ Nefrología. \\ Hospital General Universitario Gregorio Marañón. Madrid.
}

Actas Urol Esp 2005; 29 (1): 70-73

\section{RESUMEN}

TRATAMIENTO DE ADENOCARCINOMA PROSTÁTICO LOCALIZADO EN PACIENTE CON TRASPLANTE RENAL MEDIANTE ULTRASONIDO DE ALTA INTENSIDAD

Presentamos el caso de un paciente de 62 años portador de un trasplante renal (TR) que fue diagnosticado a los 6 años del implante de adenocarcinoma prostático (CP) localizado. Tras evaluar las alternativas terapéuticas se optó por el Ultrasonido de Alta Intensidad (HIFU) por vía transrectal. El resultado ha sido satisfactorio, con ingreso de 24 horas, resolución histológica y bioquímica y mínima morbilidad. No hemos hallado en la literatura referencias a la utilización de HIFU en CP en la población de trasplantados renales. Creemos que puede representar una buena alternativa terapéutica.

Palabras clave: Trasplante renal. Ultrasonido de alta intensidad. Cáncer de próstata.

\section{ABSTRACT \\ LOCALIZED PROSTATE CANCER TREATMENT IN RENAL TRANSPLANT PATIENT WITH HIGH INTENSITY FOCALIZED ULTRASOUND (HIFU)}

We report a 62 years old kidney transplant (KT) patient who was diagnosed of localized prostatic cancer (PC) after 6 years of the implant. Transrectal prostatic High Intensity Focused Ultrasound (HIFU) was applied. Results have been satisfactory, achieving pathologic and biochemical success. The discharge was completed at 24 hs, the morbidity was minimal. We have not found any reference in the literature on the appliance of HIFU in PC KT patients. We think that HIFU may represent a good alternative for these patients.

Key words: Kidney transplantation. High Intensity Focused Ultrasound. Prostate cancer.

$\mathrm{E}^{1}$ incremento de la edad media de los pacientes en lista de espera para trasplante renal se relaciona con el mayor número de diagnósticos de adenocarcinoma prostático localizado. El tratamiento de esta neoplasia es potencialmente curativo en muchos casos, y no exigiría necesariamente la suspensión del tratamiento inmunosupresor. La cirugía abierta abdominal puede ser muy dificultosa y producir más complicaciones por la fibrosis relacionada con el implante. Por otro lado, no son exactamente conocidos los efectos de las radiaciones ionizantes sobre el injerto renal. Es en estos casos donde determinadas técnicas menos invasivas -ultrasonido de alta intensidad (HIFU), crioterapia prostática- pueden resultar, de demostrar su ca- pacidad curativa, máximamente eficaces. Presentamos el caso de un paciente portador de trasplante renal todavía funcionante diagnosticado de adenocarcinoma prostático localizado en el que se utilizó como opción de tratamiento el HIFU.

\section{CASO CLÍNICO}

Paciente varón 62 años. Insuficiencia Renal Crónica terminal debido a pielonefritis crónica bilateral, en tratamiento sustitutivo con CAPD (diálisis peritoneal por intercambio) desde 1995. Trasplante Renal de donante cadáver en 1998, con nefropatía crónica del injerto posterior (creatinina actual 4,5 mg/dl). Evaluado por elevación de PSA (6 ngrs/ml) con relación PSAl/PSAt 
15,4\%. Exploración física: tacto rectal, próstata aumentada de consistencia en lóbulo derecho. Ecografía Transrectal: próstata con volumen estimado de 23 cc, nódulo hipoecoico en zona periférica de ambos lóbulos. Biopsia (Fig. 1): adenocarcinoma Score Gleason 7 en 60\% de cilindros de lóbulo derecho y cambios de PIN-3 en lóbulo izquierdo. Estudio de estadiaje con TAC y gammagrafía ósea. Se practica RTU prostática e, inmediatamente después, ultrasonido de alta intensidad prostático por vía transrectal (HIFU). Seis meses tras el tratamiento se repite la exploración ecográfica transrectal: el volumen prostático se ha reducido a 13,9 cc. La próstata muestra una imagen de ecogenicidad heterogénea (Fig. 2). Se toman 8 cilindros para estudio

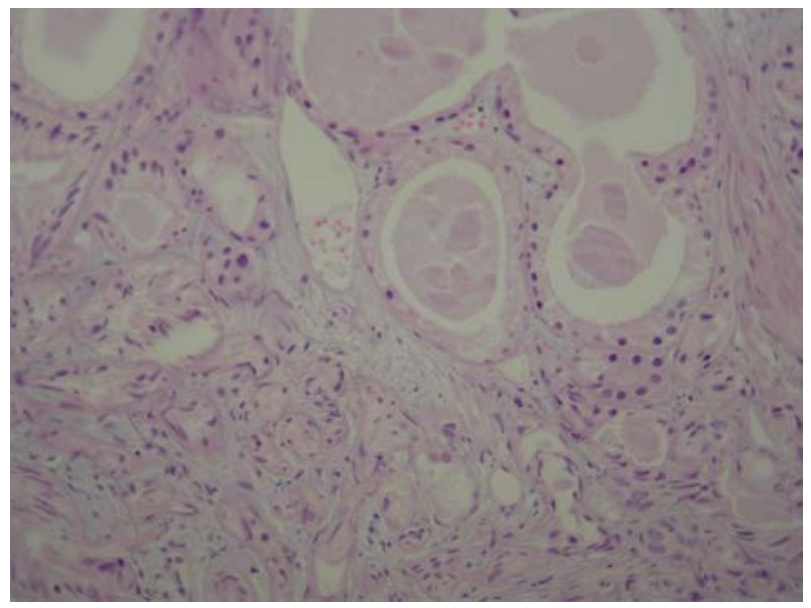

FIGURA I.

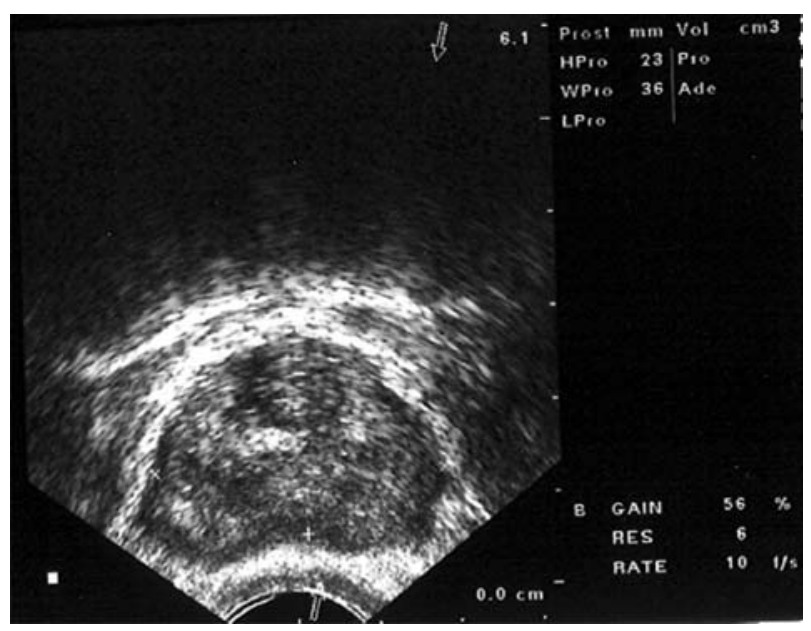

FIGURA 2. patológico, que muestran en su totalidad necrosis isquémica con focos hemorrágicos (Fig. 3). El valor del PSA se ha mantenido a 3 y 6 meses inferior a 0,04 $\mathrm{ngrs} / \mathrm{ml}$.

\section{DISCUSIÓN}

Los tumores urológicos constituyen el tipo de neoplasia no cutánea más frecuente en los pacientes con trasplante renal, representando el $34 \%$ de las mismas ${ }^{1,2}$. El cáncer de próstata (CP) constituye un pequeño porcentaje del total $(<2 \%)^{3}$. Sin embargo, el aumento de la edad media de los receptores potenciales aumenta la probabilidad de sufrir esta enfermedad ${ }^{4}$. Un alto porcentaje de receptores de primeros trasplantes renales están en el grupo de más de 45 años $^{5}$.

La realización de ecografía transrectal con biopsia prostática diagnóstica es bien tolerada en estos pacientes, con morbilidad menor y sin diferencias respecto a la población no inmunosuprimida $^{6}$. En relación a diferencias histológicas, algunos autores refieren que en el momento del diagnóstico la incidencia de CP pobremente diferenciado puede ser mayor en los pacientes con trasplante renal en comparación a la población general $^{7}$.

Desde el punto de vista terapéutico, aunque algunos autores refieren evoluciones satisfactorias en estos pacientes con la prostatectomía radical retropúbica (PRRP), las series son escasas. Una dificultad técnica de la PRRP en el paciente portador de trasplante renal es la linfa-

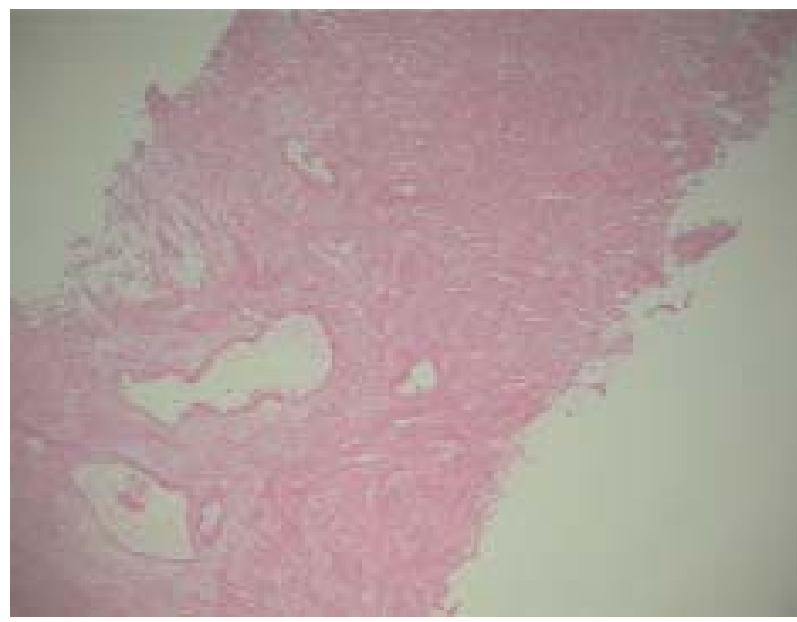

FIGURA 3. 
denectomía iliobturatriz en el lado del injerto. En estos casos puede resultar importante la utilización de nomogramas para predecir la probabilidad de enfermedad ganglionar ${ }^{8}$.

La vía perineal para la prostatectomía radical (PRP) ha sido planteada como una opción quirúrgica de menor morbilidad potencial en aquellos pacientes con baja probabilidad de sufrir extensión ganglionar por $\mathrm{su}^{\mathrm{CP}^{9}}$. La radioterapia puede inducir complicaciones potencialmente significativas y menos conocidas: estenosis ureteral del injerto, nefritis rádica. No hay series publicadas con experiencia suficiente y seguimientos largos ${ }^{9}$.

La terapia con ultrasonidos de alta intensidad (HIFU) es capaz de inducir necrosis coagulativa en cualquier medio no viscoelástico, como todos los tejidos biológicos. Las ondas de ultrasonido focalizado son emitidas desde un transductor transrectal y son absorbidas en un áreaobjetivo, produciendo efectos mecánicos, cavitación, y efectos térmicos sin inducir lesiones en los tejidos atravesados hasta alcanzar su destino $^{10}$. El objetivo principal del HIFU es producir un incremento de la temperatura a valores superiores a $70^{\circ} \mathrm{C}$ en el tejido tratado, lo que produciría un proceso de necrosis coagulativa del mismo ${ }^{11}$. Ese aumento térmico se produce en el punto tratado del órgano diana (punto focal). Sin embargo, carece de efecto en el campo comprendido entre el punto focal y la porción anterior del transductor. El flujo vascular del órgano tratado no influye en la respuesta al tratamiento. Dado que la lesión inducida -necrosis coagulativa- se produce sólo en el punto tratado manteniéndose intacto el tejido circundante, para conseguir un efecto terapéutico sobre toda la glándula prostática precisaremos de emitir radiación ultrasónica zona a zona sobre una planificación volumétrica elaborada y controlada informáticamente (Fig. 4).

Blana ${ }^{11}$ ha presentado los resultados obtenidos con un sistema Ablatherm ${ }^{\mathrm{R}}$ en una serie de 146 pacientes con un seguimiento de 5 años. Todos ellos habían sido diagnosticados de adenocarcinoma prostático estadío T1-T2 mediante biopsia, con PSA $</=15$ y Gleason-Score $</=7$. Tras 2 años de seguimiento el $87 \%$ de los pacientes mantenía un PSA < $1 \mathrm{ngr} / \mathrm{ml}$, el 93,4\% presentaba biopsias prostáticas sin presencia de cáncer.

Esta técnica se completa como terapia mínimamente invasiva, con un ingreso potencial de 24-48 horas. Puede representar una alternativa potencialmente útil en pacientes con mayor riesgo quirúrgico, como los trasplantados renales. La incidencia de complicaciones secundarias (fístulas recto-uretrales, impotencia, incotinencia urinaria) es poco significativa. La disfunción eréctil secundaria a lesión de los fascículos neurovasculares (FNV) es probablemente la consecuencia más frecuente ( $>50 \%$ casos), en los tratamientos completos. Sin embargo, dada la posibilidad de planificación ecográfica previa a la administración del ultrasonido puede respetarse el FNV del lado no afectado por tumor en casos seleccionados $^{12}$.

En nuestro caso utilizamos el HIFU con éxito, siendo la evolución del paciente hasta el momento actual satisfactoria y con control oncológico completo.

\section{CONCLUSIONES}

Presentamos el caso de un paciente portador de un trasplante renal que es diagnosticado de un CP localizado. Se utilizó

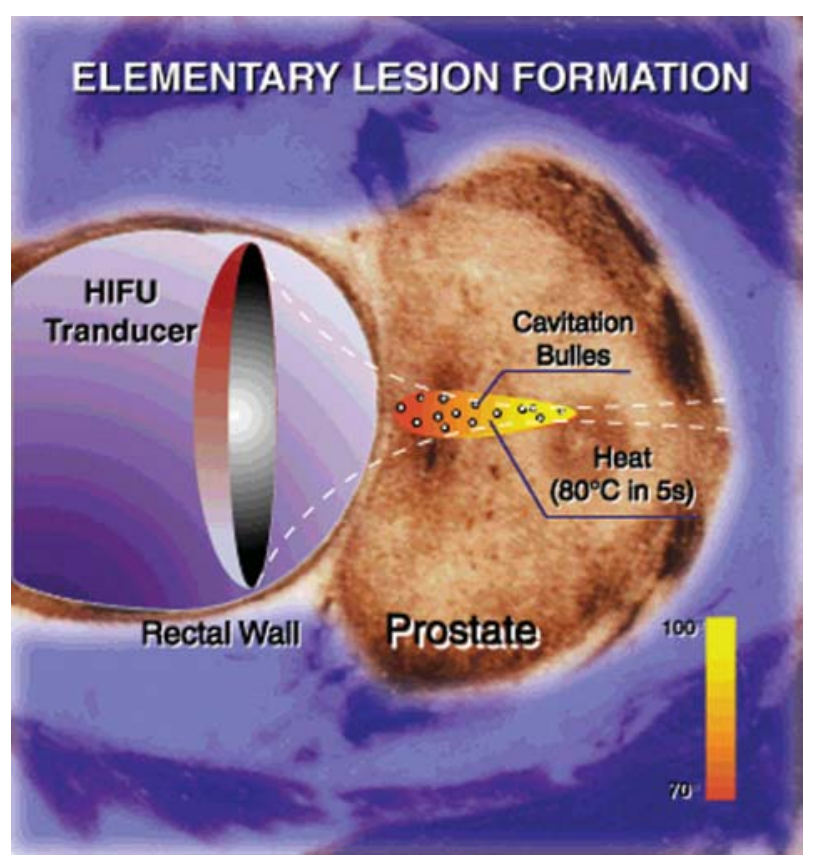

FIGURA 4. 
una alternativa terapéutica menos invasiva (Ultrasonido de Alta Intensidad) con buenos resultados iniciales, tanto bioquímicos como histológicos. La morbilidad fue mínima. Creemos en la utilidad potencial de estas técnicas en ciertos grupos de pacientes de mayor riesgo quirúrgico. Sin embargo, se necesitan estudios prospectivos, randomizados y con seguimientos a más de 5 años para establecer la solidez definitiva de estas técnicas.

\section{Agradecimientos}

Al Prof. Stefan Thüroff por sus enseñanzas así como por su amabilidad permitiéndonos utilizar una de sus diapositivas (Fig. 4).

\section{REFERENCIAS}

1. Parker SL, Tong T, Bolden S. Cancer Statistics, 1996. CA Cancer J Clin 1996;65:5-27.

2. Khauli RB. Genitourinary malignancies in organ transplant recipients. Semin Urol 1994;12:224-232.

3 . Penn I. The problem of cancer in organ transplant recipients: an overview. Transpl Sci 1994;4:23-32.

4. Konety BR, Tewari A, Howard RJ, Barry JM, Hodge EE, Taylor R, Jordan ML. Prostate Cancer in the post-transplant population. Urology 1998;52:428-432.

5. Cecka JM, Terasaki PI. The UNOS scientific transplant registry, in Cecka JM, Terasaki PI (Eds): Clinical
Transplants. Los Angeles, UCLA Tissue Typing Laboratory, 1994:1.

6. Wammack R, Djavan B, Remzi M, Susani M, Marberger M. Morbidity of transrectal ultrasound-guided prostate needle biopsy in patients receiving inmunosupression. Urology 2001;58:1004-1007.

7. Lechevallier E, Cormier L, Barrou B, Benoit G, Bensadoun H, Boudjema K, Descottes JL, Doré B, Guy L, Malavaud B, Martín X, Petit J, Salomón L. Prostate Cancer in Renal Transplant Recipients. Transpl Proc 2002;34:722.

8. Ciancio G, Antun RA, Norberg DG. Prostate Cancer after Heart transplantation. J Urol 1995; 153:158-160.

9. Yiou R, Salomon L, Colombel M, Patard JJ, Chopin D, Abbou CC. Perineal approach to radical prostatectomy in kidney transplant recipients with localized prostate cancer. Urology 1999;53:822-824.

10. Huber P, Debus J, Jenne J. Thrapeutic Ultrasound in tumor therapy: principles, applications and new developments. Radiologe 1996;36:64-71.

11. Blana A, Walter B, Rogenhofer S, Wieland WF. HighIntensity Focused Ultrasound for the treatment of localized prostate cancer: 5-year experience. Urology 2004;63:297300.

12. Thuroff S. Comunicación Personal. 2003-2004.

\section{Dr. E. Lledó García}

Servicio de Urología

Hospital General Universitario Gregorio Marañón

Doctor Esquerdo, 46

28007 Madrid

(Trabajo recibido el 16 junio de 2004) 